\title{
Gestión de turismo gastronómico: identificación del mercado potencial en Canarias
}

\author{
Daniel Dorta-Afonso* \\ Universidad de Las Palmas de Gran Canaria (Spain) \\ Hugo Padrón-Ávila** \\ Universidad de La Laguna (España)
}

\begin{abstract}
Resumen: La coyuntura turística actual ha derivado en que los turistas demandan experiencias únicas y auténticas. Sin embargo, la provisión de dichas experiencias no es una tarea fácil para los destinos y las empresas turísticas. La literatura sugiere que la gastronomía, como parte de la cultura de las comunidades que visitan los turistas tiene el potencial de ofrecer a los turistas esa autenticidad y singularidad demandada. Así, nos basamos en la literatura sobre turismo gastronómico para identificar su potencial como factor motivador en la organización del viaje y como potenciador de la satisfacción de los turistas. El objetivo de este estudio es analizar cuáles son las características de los visitantes más propensos a mostrarse más interesados por la gastronomía de Canarias. Por lo tanto, en el presente estudio identificamos el perfil del turista para los cuales la gastronomía juega un papel esencial motivador a la hora de viajar. Para ello hemos utilizado las respuestas de los turistas a la Encuesta sobre Gasto Turístico y llevado a cabo análisis de regresión. Los resultados sugieren que existen diferencias en el perfil sociodemográfico, motivacional y en las características del viaje de este segmento particular de turistas motivados por la gastronomía. Las conclusiones del estudio señalan que el segmento de turistas motivado por la gastronomía tiene un tamaño considerable en Canarias y es necesario mejorar la satisfacción de estos turistas ya que son más propensos a gastar más en el destino que el visitante medio.
\end{abstract}

Palabras Clave: Gastronomía; Turismo gastronómico; Segmentación de turistas; Turista gastronómico; Motivación de turistas.

\section{Gastronomic tourism management: identifying the potential market in the Canaries}

Abstract: The current situation of tourism has derived towards tourists demanding authentic and unique tourism experiences. However, providing tourists with such experiences is not a simple task for tourism firms and destinations. The literature suggests that gastronomy, as part of the culture of the communities tourists visit, has the potential to offer the uniqueness and authenticity demanded. We therefore build on food tourism literature to identify its potential as a motivational factor as well as an enhancer of tourists' satisfaction. The aim of this study is to analyzs the characteristics of those visitors most likely to be interested in the consumption of typical local gastronomical products when visiting the Canary Islands. Consequently, in the present study we identify the profile of tourists for whom gastronomy plays an essential motivational role when travelling. To do so, we have used the answers given by tourists on the Tourism Expenditure Survey and conducted regression analysis. The results suggest that there are differences in the socio-demographic profile of gastronomic tourists to Canary Islands, their motivations and also the characteristics of their trips. The conclusions of the study indicate that the segment of tourists motivated by gastronomy is considerable in the Canary Islands and it is necessary to improve the satisfaction of these tourists since they are more likely to spend more in the destination than the average visitor.

Keywords: Gastronomy; Food tourism; Tourist segmentation; Food tourists; Tourist motivations.

\footnotetext{
* Universidad de Las Palmas de Gran Canaria (Spain); E-mail: daniel.dorta@ulpgc.es; https://orcid.org/0000-0002-9955-1202

**Universidad de La Laguna (España); E-mail: hpadrona@ull.edu.es; https://orcid.org/0000-0001-6181-4698
} 


\section{Introducción}

El turismo es la principal actividad económica de muchas regiones y está ampliamente aceptado que su fin último es mejorar la calidad de vida de los habitantes de una región determinada (e.g., Dwyer \& Kim, 2003; Ritchie \& Crouch, 2003). De hecho, las últimas cifras económicas corroboran que efectivamente el turismo contribuye a un 10,4\% del PIB mundial, con una tasa de crecimiento mayor al del total de la economía, y que además supone el 3,8\% de los empleados a nivel global (WTTC, 2019). Sin embargo, mantener niveles altos de competitividad no es una tarea fácil para los destinos y empresas del sector.

En esta línea, existe un determinado consenso en que los destinos turísticos pueden mantener y mejorar sus niveles de competitividad mediante la provisión a los turistas de experiencias satisfactorias y memorables (Dorta-Afonso \& Hernández-Martín, 2015; Ritchie \& Crouch, 2003). Sin embargo, la búsqueda de autenticidad y de experiencias cada vez más únicas por parte de los turistas se ha ido convirtiendo de manera progresiva en uno de los principales retos para empresas y destinos turísticos de cara a la provisión de experiencias satisfactorias mencionada (Wang, 1999). En este sentido, la gastronomía ha sido señalada en numerosos estudios como una parte esencial de la cultura local de los destinos turísticos y por lo tanto tiene la capacidad, por sí misma, de ofrecer a los turistas experiencias nuevas, agradables y auténticas (Berbel-Pineda, Palacios-Florencio, Ramírez-Hurtado, \& Santos-Roldán, 2019; S. Kim, Park, \& Lamb, 2019; Lin \& Mao, 2015; Tikkanen, 2007). Así, no es de extrañar que en los últimos años haya incrementado el interés por parte de la comunidad científica en el turismo gastronómico, o en la gastronomía como parte esencial de las experiencias en destino (e.g., Chavarria \& Phakdee-auksorn, 2017; Cohen \& Avieli, 2004; López-Guzmán, Uribe Lotero, Perez Galvez, \& Rios Rivera, 2017).

El presente artículo está organizado de la siguiente forma: tras la introducción presentamos la revisión de la literatura y los objetivos del estudio. Posteriormente, pasamos a presentar la metodología del estudio incluyendo la descripción de la base de datos utilizada y la estrategia de análisis estadísticos que se ha seguido. Por último, se presentan los resultados del estudio antes de proceder a la discusión de los resultados y las principales conclusiones que se derivan de esta investigación.

\section{Revisión de la literatura sobre gastronomía y turismo}

El turismo gastronómico se define como la visita de los turistas a productores de comida, festivales orientados a la gastronomía, restaurantes o localizaciones para los cuales la degustación de productos gastronómicos es un factor motivacional a la hora de elegir el destino turístico y las características del viaje (Chen \& Huang, 2016; Hall, Sharples, Mitchell, Macionis, \& Cambourne, 2004). Con vistas a delimitar el concepto de turismo gastronómico, es importante destacar que el consumo de gastronomía es una actividad llevada a cabo por la totalidad de los turistas que visitan un destino y que, por lo tanto, cualquier visita a un restaurante no debería de ser considerado como turismo gastronómico. Además, y en aras de pulir la definición, también hay que destacar que los ofertantes de gastronomía no tienen por qué cumplir ninguna característica específica, como la posesión de una estrella Michelin, sino que el consumo de platos típicos en cualquier restaurante local puede ser considerado como turismo gastronómico, siempre que haya sido uno de los motivadores esenciales del viaje (Berbel-Pineda et al., 2019).

El debate con respecto al tamaño del segmento de mercado que tiene un interés especial por la gastronomía, así como su importancia como factor motivador y su impacto económico está actualmente abierto (e.g., Berbel-Pineda et al., 2019; López-Guzmán et al., 2017; McKercher, Okumus, \& Okumus, 2008). Por una parte, existen investigadores que claman activamente que la importancia del turismo gastronómico está en auge bajo la premisa de que cada vez más personas viajan exclusivamente motivados por los atributos gastronómicos de un determinado destino (Quan \& Wang, 2004). En este sentido, se entiende que la gastronomía es un elemento motivador por sí mismo y por lo tanto tiene la capacidad de atraer a turistas que viajan en busca de la autenticidad que deriva de la degustación de este tipo de productos (Sánchez-Cañizares \& López-Guzmán, 2012; Wijaya, King, Nguyen, \& Morrison, 2013). Por otro lado, otros estudios llaman la atención sobre la posible sobreestimación que puede haber en los estudios que abogan por la importancia del turismo gastronómico y, de hecho, sugieren que la realidad del mercado puede ser muy diferente y que en términos prácticos el segmento de mercado motivado exclusivamente por la gastronomía a la hora de viajar no sea tan importante en términos relativos (McKercher et al., 2008).

Sea como fuere, sí que existe un amplio acuerdo en que la gastronomía de un destino, como parte esencial de su cultura e identidad puede mejorar la competitividad de empresas y destinos independientemente de la demanda que genera por sí misma (Berbel-Pineda et al., 2019). Esto es debido a que ya sea un factor motivador principal o accesorio puede influir en los patrones de consumo de los turistas (S. Kim et al., 2019). De hecho, los estudios previos indican que la gastronomía constituye una 
de las principales fuentes de gasto independientemente del rol motivacional de la misma en la elección del destino (Chen \& Huang, 2016). Así, la gastronomía tiene, definitivamente, el potencial de añadir valor a la experiencia de los viajeros, y por lo tanto, es un factor determinante de la satisfacción de los turistas con sus vacaciones (Chen \& Huang, 2016; Kim, Goh, \& Yuan, 2010). Sin embargo, la mayoría de los estudios se han centrado en situar los atributos gastronómicos del destino como herramienta estratégica en el marketing de los destinos (Du Rand \& Heath, 2006), mientras que pocos estudios se han llevado a cabo desde el punto de vista de la demanda. Así, es necesario profundizar en la literatura sobre turismo gastronómico sobre la importancia de conocer el perfil del turista gastronómico (Chen \& Huang, 2016; Ryu \& Jang, 2006).

\section{Objetivo, propuesta e innovación del estudio realizado}

En el presente estudio pretendemos dar respuesta a varias preguntas que están actualmente sin respuesta en la literatura académica. En primer lugar queremos cuantificar la importancia del segmento de turistas que consideran la gastronomía como elemento motivador a la hora de planificar sus vacaciones, dando respuesta así a la controversia planteada con respecto a la importancia del mercado de turismo gastronómico (McKercher et al., 2008; Sánchez-Cañizares \& López-Guzmán, 2012). Además, y debido a que la mayoría de los estudios se plantean desde la perspectiva de la oferta, con un enfoque mayoritario en el potencial de la gastronomía como elemento de marketing de destinos, la literatura no se ha centrado en caracterizar el perfil del turista que viaja en base a esta motivación determinada (Du Rand \& Heath, 2006; Mason \& Paggiaro, 2012). Así, con un enfoque desde el punto de vista de la demanda, damos respuesta a la caracterización necesaria del perfil del turista cuya motivación de viaje es la gastronomía (Chen \& Huang, 2016). Para ello, hemos acudido a la Encuesta sobre Gasto Turístico (EGT) realizada por el Instituto Canario de Estadística (ISTAC) que recoge el perfil sociodemográfico de los turistas que visitan el destino Islas Canarias, así como los elementos motivacionales que inspiraron el viaje, las características de los viajes realizados, el gasto que han llevado tanto en origen como en destino, así como su satisfacción y lealtad con el destino.

La realización de esta investigación tiene importantes contribuciones teóricos y prácticas. En primer lugar damos respuesta a la problemática planteada con respecto a la cuantificación real del segmento de mercado que viaja motivado por la gastronomía (McKercher et al., 2008). Además, con la caracterización del perfil del turista gastronómico, complementamos los estudios previos que se han centrado en el estudio de la oferta (Du Rand \& Heath, 2006). Así, nuestros resultados pueden ser utilizados por las empresas de restauración, así como por las asociaciones gestoras de los destinos de cara a mejorar sus propuestas de valor gastronómicas y para mejorar el enfoque de sus campañas de marketing.

\section{Metodología}

En este estudio se emplean dos herramientas de análisis diferentes para conocer cuáles son las características de los turistas que visitan Canarias con el objetivo de realizar un viaje de turismo gastronómico. En primer lugar, se ha realizado un análisis mediante tablas de contingencia para conocer cuáles son las características del turista que da mayor importancia a la gastronomía a la hora de elegir el destino de su viaje. Tras ello, se ha aplicado una regresión lineal para conocer cuáles son las variables significativas que explican por qué los turistas con estas características eligen Canarias como destino. La investigación previa apunta a otras técnicas de análisis que podrían haberse aplicado para estudiar las elecciones de los turistas, como modelos de elección discreta (Padrón-Ávila \& Hernández Martín, 2019a, 2019b) o ecuaciones estructurales (Quan \& Wang, 2004). Sin embargo, en el caso de los datos empleados para el análisis, la variable dependiente presenta una escala Likert que no permite que sea utilizada como variable dependiente en modelos de elección discreta y, al existir múltiples formatos de variables independientes (no solo Likert), tampoco pueden aplicarse ecuaciones estructurales. Por ello, se ha decidido realizar una regresión lineal, que permite combinar estos tipos de variables (Rasoolimanesh, Ali, \& Jaafar, 2018).

Para la realización de este estudio se ha utilizado la base de datos de la EGT del ISTAC. En concreto, se han utilizado los datos recopilados durante 2018. En la encuesta, se pregunta a los turistas por sus características demográficas, motivaciones, medio usado para conocer el destino, características del viaje, acompañantes, etc. La base de datos empleada contiene las respuestas de 37.904 turistas que fueron encuestados en los aeropuertos canarios que cuentan con vuelos interregionales o internacionales, antes de regresar a sus lugares de origen (ISTAC, 2018).

Para procesar los datos, la función para crear tablas dinámicas de Excel fue utilizada para realizar tablas de contingencia, mientras que el software SPSS fue empleado para realizar una regresión lineal y 
conocer cuáles son las variables relacionadas con que los turistas gastronómicos visiten el archipiélago. $\mathrm{Al}$ realizar el estudio mediante tablas de contingencias, solo se han considerado variables demográficas de los turistas que permitan establecer las diferencias generales entre los turistas gastronómicos y aquellos que no lo son, tal como se realizó en el estudio de Padrón-Ávila \& Hernández-Martín (2017). Sin embargo, hemos considerado una variedad mayor de variables al realizar la regresión lineal. Se han tenido en cuenta diferentes variables relativas a las características demográficas de los turistas, sus motivaciones, canales para conocer la existencia de Canarias, características del viaje, número y tipo de acompañantes, gasto durante el viaje, actividades realizadas, y satisfacción y fidelización con el destino.

Para realizar ambos análisis, la variable motivacional relacionada con elegir el destino por su gastronomía ha sido utilizada como variable dependiente. Se trata de una variable con formato de escala Likert de 4 ítems, donde el valor 1 significa que la gastronomía no influyó en la elección del viaje y el 4 que fue un aspecto fundamental para elegir el mismo. En la Tabla 1 puede observarse el peso relativo de cada una de las variables motivacionales incluidas en la encuesta del ISTAC. Así, cuando el coeficiente es positivo en la regresión, un mayor valor de la variable dependiente estará vinculado a un mayor valor de la variable independiente. Por ejemplo, si la variable "Edad" muestra un coeficiente positivo, cuanto mayor sea la edad, mayor tenderá a ser la probabilidad de elegir el destino por su gastronomía. En el caso de variables binarias como los canales para conocer el destino o los acompañantes durante el viaje; se les ha otorgado el valor 1 cuando el visitante ha marcado en la encuesta la casilla correspondiente a cada ítem (como viajar solo o conocer el destino por amigos) y el valor 0 se ha dado cuando la variable no ha sido marcada. En el caso de la variable "Sexo", el valor 0 ha sido dado a los turistas que indicaron ser hombres y el 1 a las mujeres.

\section{Tabla 1: Importancia relativa de los factores motivacionales de elección de Canarias como destino}

\begin{tabular}{|l|c|c|c|c|}
\hline Motivación & Nada relevante & Poco relevante & Relevante & Muy relevante \\
\hline Clima & $1,08 \%$ & $2,75 \%$ & $19,02 \%$ & $77,15 \%$ \\
\hline Playa & $8,87 \%$ & $22,02 \%$ & $32,11 \%$ & $37,00 \%$ \\
\hline Mar & $5,86 \%$ & $17,17 \%$ & $33,50 \%$ & $43,46 \%$ \\
\hline Paisajes & $7,42 \%$ & $21,91 \%$ & $36,60 \%$ & $34,07 \%$ \\
\hline Medioambiente & $5,88 \%$ & $19,10 \%$ & $43,15 \%$ & $31,87 \%$ \\
\hline Senderos & $49,94 \%$ & $25,22 \%$ & $14,65 \%$ & $10,19 \%$ \\
\hline Alojamiento turístico & $9,31 \%$ & $13,47 \%$ & $37,21 \%$ & $40,01 \%$ \\
\hline Patrimonio histórico & $30,81 \%$ & $37,32 \%$ & $24,42 \%$ & $7,45 \%$ \\
\hline Oferta cultural & $26,84 \%$ & $39,04 \%$ & $26,60 \%$ & $7,52 \%$ \\
\hline Diversión & $14,30 \%$ & $27,61 \%$ & $36,66 \%$ & $21,43 \%$ \\
\hline Ocio nocturno & $49,12 \%$ & $28,71 \%$ & $14,42 \%$ & $7,75 \%$ \\
\hline Oferta comercial & $32,69 \%$ & $33,99 \%$ & $23,79 \%$ & $9,53 \%$ \\
\hline Gastronomía & $14,64 \%$ & $23,89 \%$ & $39,14 \%$ & $22,32 \%$ \\
\hline Viaje sencillo & $9,89 \%$ & $16,66 \%$ & $40,26 \%$ & $33,18 \%$ \\
\hline Seguridad & $4,82 \%$ & $9,79 \%$ & $35,28 \%$ & $50,11 \%$ \\
\hline Tranquilidad & $3,16 \%$ & $10,66 \%$ & $39,80 \%$ & $46,38 \%$ \\
\hline Pertenencia a Europa & $19,09 \%$ & $16,76 \%$ & $29,37 \%$ & $34,78 \%$ \\
\hline Precio & $4,86 \%$ & $17,28 \%$ & $41,68 \%$ & $36,18 \%$ \\
\hline Exotismo & $27,55 \%$ & $34,40 \%$ & $26,75 \%$ & $11,30 \%$ \\
\hline Autenticidad & $13,97 \%$ & $28,25 \%$ & $37,66 \%$ & $20,12 \%$ \\
\hline & & & & \\
\hline
\end{tabular}

Fuente: Elaboración propia en base a los datos de la EGT 


\section{Resultados}

En primer lugar, se pretendía conocer si el sexo era una variable relevante para explicar por qué los turistas elegían Canarias como destino para su viaje. Estudios previos han mostrado que el sexo no es una variable relevante que determine la elección de actividades por parte de los turistas que visitan el archipiélago (Padrón-Ávila \& Hernández-Martín, 2019b). Sin embargo, esto no había sido probado al estudiar la relación entre esta variable y la elección del destino para disfrutar de su gastronomía. Los resultados aparecen en la Tabla 2 y no muestran grandes diferencias entre hombres y mujeres. Los hombres parecen ser algo más propensas a emitir valoraciones intermedias (algo o bastante), mientras que las mujeres parecen presentar una tendencia un poco mayor a marcar nada o mucho (los extremos). Además, los hombres señalan, de media, que son un poco más propensos a elegir el destino por su gastronomía.

Tabla 2: Turistas gastronómicos en función de su sexo

\begin{tabular}{|l|c|c|c|c|c|}
\hline \multirow{2}{*}{ Edad } & \multicolumn{4}{|c|}{ Elegir Canarias por la gastronomía } & \multirow{2}{*}{$\begin{array}{c}\text { Valoración } \\
\text { media }\end{array}$} \\
\cline { 2 - 5 } & Nada & Algo & Bastante & Mucho & 2,70 \\
\hline Hombre & $14 \%$ & $24 \%$ & $41 \%$ & $22 \%$ & 2,68 \\
\hline Mujer & $15 \%$ & $24 \%$ & $38 \%$ & $23 \%$ & \\
\hline
\end{tabular}

Fuente: Elaboración propia en base a los datos de la EGT

Tras realizar este análisis, se creó una segunda tabla de contingencia para analizar el impacto de la edad en la elección de Canarias como destino de turismo gastronómico. Los resultados del análisis se presentan en la Tabla 3 y parecen indicar que, en general, los turistas de mayor edad tienen una tendencia mayor a elegir el destino para practicar turismo gastronómico que los turistas más jóvenes. Esta tendencia es más elevada en el segmento de turistas que tiene entre 56 y 65 años. De hecho, a pesar de que, en general, los turistas gastronómicos están más motivados a elegir Canarias como destino conforme incrementa su edad, a partir del rango 56-65, parece que esta tendencia comienza a reducirse. De hecho, los turistas de 76 o más años parecen ser menos propensos a elegir Canarias por su gastronomía que los turistas entre 46 y 55 años.

Tabla 3: Turistas gastronómicos en función de su edad

\begin{tabular}{|c|c|c|c|c|c|}
\hline \multirow{2}{*}{ Edad } & \multicolumn{4}{|c|}{ Elegir Canarias por la gastronomía } & \multirow{2}{*}{$\begin{array}{c}\text { Valoración } \\
\text { media }\end{array}$} \\
\cline { 2 - 5 } & Nada & Algo & Bastante & Mucho & 2,49 \\
\hline $16-25$ & $24 \%$ & $25 \%$ & $30 \%$ & $21 \%$ & 2,66 \\
\hline $26-35$ & $16 \%$ & $24 \%$ & $36 \%$ & $24 \%$ & 2,67 \\
\hline $36-45$ & $15 \%$ & $25 \%$ & $39 \%$ & $21 \%$ & 2,75 \\
\hline $46-55$ & $12 \%$ & $23 \%$ & $42 \%$ & $22 \%$ & 2,78 \\
\hline $56-65$ & $11 \%$ & $23 \%$ & $43 \%$ & $23 \%$ & 2,77 \\
\hline $66-75$ & $12 \%$ & $22 \%$ & $43 \%$ & $23 \%$ & 2,73 \\
\hline 76 o más & $13 \%$ & $24 \%$ & $41 \%$ & $22 \%$ & \\
\hline
\end{tabular}

Fuente: Elaboración propia en base a los datos de la EGT

El último análisis mediante una tabla de contingencia realizado ha consistido en comparar la elección de Canarias como destino de turismo gastronómico en función del país de procedencia de los turistas. Los resultados alcanzados se presentan en la Tabla 4. Los resultados apuntan a grandes diferencias en función de la nacionalidad del visitante. Los turistas residentes en Islandia son los menos propensos a elegir Canarias como destino por su gastronomía, mientras que los senegaleses son los más propensos a hacerlo, existiendo 
una diferencia de más de un punto de media (sobre 4) entre ellos. Sin contar a los turistas residentes en Senegal, los residentes en Rumania son los turistas para los que la gastronomía canaria tiene un mayor peso en su elección del destino. Los datos indican que los turistas residentes en países africanos son, en general, los más propensos a viajar a Canarias por su gastronomía. Sin embargo, los países del norte europeo tienden a mostrar la propensión más baja a haber elegido el destino debido a su gastronomía.

Tabla 4: Turistas gastronómicos en función de su país de residencia

\begin{tabular}{|c|c|c|c|c|c|}
\hline \multirow{2}{*}{ País } & \multicolumn{4}{|c|}{ Elegir Canarias por la gastronomía } & \multirow{2}{*}{$\begin{array}{l}\text { Valoración } \\
\text { media }\end{array}$} \\
\hline & Nada & Algo & Bastante & Mucho & \\
\hline Alemania & $11 \%$ & $26 \%$ & $41 \%$ & $21 \%$ & 2,73 \\
\hline Austria & $12 \%$ & $17 \%$ & $45 \%$ & $27 \%$ & 2,86 \\
\hline Bélgica & $8 \%$ & $22 \%$ & $44 \%$ & $26 \%$ & 2,88 \\
\hline Chequia & $9 \%$ & $18 \%$ & $44 \%$ & $29 \%$ & 2,93 \\
\hline Dinamarca & $23 \%$ & $28 \%$ & $33 \%$ & $17 \%$ & 2,44 \\
\hline España & $11 \%$ & $26 \%$ & $40 \%$ & $23 \%$ & 2,75 \\
\hline Estonia & $3 \%$ & $36 \%$ & $40 \%$ & $21 \%$ & 2,78 \\
\hline Finlandia & $14 \%$ & $29 \%$ & $44 \%$ & $14 \%$ & 2,57 \\
\hline Francia & $8 \%$ & $28 \%$ & $45 \%$ & $18 \%$ & 2,74 \\
\hline Hungría & $10 \%$ & $19 \%$ & $48 \%$ & $24 \%$ & 2,86 \\
\hline Irlanda & $19 \%$ & $18 \%$ & $33 \%$ & $30 \%$ & 2,75 \\
\hline Islandia & $25 \%$ & $34 \%$ & $28 \%$ & $13 \%$ & 2,29 \\
\hline Italia & $10 \%$ & $28 \%$ & $43 \%$ & $20 \%$ & 2,73 \\
\hline Letonia & $27 \%$ & $9 \%$ & $32 \%$ & $32 \%$ & 2,68 \\
\hline Lituania & $17 \%$ & $0 \%$ & $50 \%$ & $33 \%$ & 3,00 \\
\hline Luxemburgo & $8 \%$ & $14 \%$ & $44 \%$ & $34 \%$ & 3,04 \\
\hline Marruecos & $6 \%$ & $22 \%$ & $36 \%$ & $36 \%$ & 3,02 \\
\hline Mauritania & $12 \%$ & $16 \%$ & $32 \%$ & $40 \%$ & 3,00 \\
\hline Noruega & $18 \%$ & $25 \%$ & $39 \%$ & $18 \%$ & 2,58 \\
\hline Países Bajos & $22 \%$ & $29 \%$ & $36 \%$ & $13 \%$ & 2,40 \\
\hline Polonia & $8 \%$ & $18 \%$ & $43 \%$ & $31 \%$ & 2,97 \\
\hline Portugal & $11 \%$ & $32 \%$ & $39 \%$ & $18 \%$ & 2,65 \\
\hline Reino Unido & $20 \%$ & $21 \%$ & $36 \%$ & $23 \%$ & 2,62 \\
\hline Rumania & $3 \%$ & $16 \%$ & $46 \%$ & $35 \%$ & 3,14 \\
\hline Rusia & $9 \%$ & $19 \%$ & $36 \%$ & $36 \%$ & 2,99 \\
\hline Senegal & $0 \%$ & $0 \%$ & $50 \%$ & $50 \%$ & 3,50 \\
\hline Suecia & $14 \%$ & $21 \%$ & $43 \%$ & $22 \%$ & 2,72 \\
\hline Suiza & $12 \%$ & $25 \%$ & $30 \%$ & $32 \%$ & 2,82 \\
\hline Venezuela & $11 \%$ & $33 \%$ & $22 \%$ & $33 \%$ & 2,78 \\
\hline
\end{tabular}

Fuente: Elaboración propia en base a los datos de la EGT

Por último, se presenta la Tabla 5 en la que pueden observarse los resultados obtenidos mediante la regresión lineal para las variables empleadas. Se utilizaron 86 variables independientes para conocer 
cómo se vinculan las mismas con la elección de Canarias como destino gastronómico. De entre las mismas, 37 mostraron no ser significativas para el estudio realizado, 5 mostraron una ligera tendencia a influir en la elección de Canarias con el objetivo de disfrutar de su gastronomía, 11 fueron significativas y el resto mostraron tener un nivel de significatividad muy elevado. No pudo incluirse la nacionalidad como variable demográfica en el análisis porque se trataba de una variable categórica en lugar de ser numérica. En la tabla, se han dividido las variables por categorías para facilitar su análisis.

Tabla 5: Resultados de la regresión lineal estableciendo cuáles son las variables que explican por qué los turistas eligen Canarias como destino gastronómico.

\begin{tabular}{|c|c|c|}
\hline Variable & Coeficiente & p value \\
\hline \multicolumn{3}{|l|}{ Demográficas } \\
\hline Sexo & $-0,009$ & $0,000 * * *$ \\
\hline Edad & 0,006 & $0,000 * * *$ \\
\hline Nivel educativo & 0,055 & $0,000 * * *$ \\
\hline Ingresos & 0,026 & $0,000 * * *$ \\
\hline Personas en el hogar & $-0,018$ & $0,000 * * *$ \\
\hline \multicolumn{3}{|c|}{ Motivos de elección del Canarias } \\
\hline Clima & $-0,003$ & 0,779 \\
\hline Playa & 0,011 & 0,169 \\
\hline Mar & 0,036 & $0,000 * * *$ \\
\hline Paisajes & 0,030 & $0,000^{* * *}$ \\
\hline Entorno ambiental & 0,033 & $0,000 * * * *$ \\
\hline Senderismo & 0,023 & $0,000 * * *$ \\
\hline Oferta alojativa & 0,037 & $0,000 * * *$ \\
\hline Patrimonio histórico & 0,059 & $0,000 * * *$ \\
\hline Oferta cultural & 0,068 & $0,000 * * *$ \\
\hline Diversión & 0,025 & $0,000 * * *$ \\
\hline Ocio nocturno & 0,040 & $0,000 * * *$ \\
\hline Oferta comercial & 0,155 & $0,000 * * *$ \\
\hline Facilidad del viaje & 0,164 & $0,000 * * *$ \\
\hline Seguridad & 0,076 & $0,000 * * *$ \\
\hline Tranquilidad & 0,075 & $0,000 * * *$ \\
\hline Estar en Europa & $-0,012$ & $0,016^{* *}$ \\
\hline Precio & $-0,025$ & $0,001^{* * *}$ \\
\hline Exotismo & 0,022 & $0,001^{* * *}$ \\
\hline Autenticidad & 0,109 & $0,000 * * *$ \\
\hline \multicolumn{3}{|c|}{ Canales para conocer el destino } \\
\hline Visitas previas & $-0,001$ & 0,780 \\
\hline Amigos & 0,002 & 0,417 \\
\hline Redes sociales & 0,003 & 0,188 \\
\hline Medios de comunicación & $-0,001$ & 0,942 \\
\hline Revistas turismo & $-0,010$ & $0,003^{* * *}$ \\
\hline
\end{tabular}




\begin{tabular}{|c|c|c|}
\hline Variable & Coeficiente & p value \\
\hline Blogs & 0,015 & $0,001^{* * *}$ \\
\hline Televisión & $-0,021$ & $0,084^{*}$ \\
\hline Intermediario & $-0,000$ & 0,989 \\
\hline Asociaciones & 0,013 & 0,385 \\
\hline Otros & 0,015 & $0,043^{* *}$ \\
\hline \multicolumn{3}{|c|}{ Características del viaje } \\
\hline Paquete turístico & 0,006 & 0,320 \\
\hline Pernoctaciones & $-0,002$ & $0,006^{* * *}$ \\
\hline Categoría alojamiento & 0,008 & $0,039 * *$ \\
\hline Coste paquete & $-0,006$ & 0,290 \\
\hline \multicolumn{3}{|l|}{ Acompañantes } \\
\hline Solo & $-0,002$ & 0,654 \\
\hline Pareja & 0,006 & $0,059^{*}$ \\
\hline Hijos & $-0,003$ & 0,386 \\
\hline Otros familiares & $-0,003$ & 0,484 \\
\hline Amigos & $-0,002$ & 0,551 \\
\hline Compañeros de trabajo & 0,003 & 0,874 \\
\hline Viaje organizado & $-0,004$ & 0,746 \\
\hline Número personas & 0,006 & 0,224 \\
\hline \multicolumn{3}{|l|}{ Gasto durante el viaje } \\
\hline Total & 0,000 & $0,000 * * *$ \\
\hline Alojamiento & 0,001 & 0,266 \\
\hline Vuelo & 0,002 & $0,058^{*}$ \\
\hline Taxi & 0,001 & 0,185 \\
\hline Alquiler coche & 0,000 & 0,930 \\
\hline Transporte público & 0,001 & 0,534 \\
\hline Supermercados & 0,000 & 0,508 \\
\hline Restaurantes & 0,002 & $0,000^{* * *}$ \\
\hline Excursiones & 0,000 & 0,924 \\
\hline Deportes & $-0,001$ & $0,096^{*}$ \\
\hline Actividades culturales & 0,001 & 0,758 \\
\hline Museos & $-0,001$ & 0,422 \\
\hline Parques de ocio & $-0,001$ & 0,209 \\
\hline Discotecas & 0,000 & 0,660 \\
\hline Salud & 0,001 & 0,499 \\
\hline Suvenires & 0,000 & 0,833 \\
\hline Bienes inmuebles & $-0,007$ & $0,018^{* *}$ \\
\hline Otras compras & 0,002 & 0,263 \\
\hline Farmacia & 0,000 & 0,950 \\
\hline
\end{tabular}




\begin{tabular}{|c|c|c|}
\hline Variable & Coeficiente & p value \\
\hline Otros & 0,001 & $0,042^{* *}$ \\
\hline \multicolumn{3}{|l|}{ Actividades realizadas } \\
\hline Playa & $-0,009$ & $0,001 * * *$ \\
\hline Piscina & $-0,005$ & $0,042^{* *}$ \\
\hline Pasear & 0,002 & 0,424 \\
\hline Visitar otra isla & $-0,005$ & $0,043^{* *}$ \\
\hline Excursiones organizadas & $-0,009$ & $0,002^{* * *}$ \\
\hline Excursiones marítimas & $-0,001$ & 0,751 \\
\hline Astronomía & 0,012 & $0,021^{* *}$ \\
\hline Museos & $-0,011$ & $0,003^{* * *}$ \\
\hline Cultura popular & 0,011 & $0,000 * * *$ \\
\hline Gastronomía & 0,054 & $0,000 * * *$ \\
\hline Parques de ocio & $-0,009$ & $0,005^{* * *}$ \\
\hline Ocio & $-0,014$ & $0,000 * * *$ \\
\hline Belleza & 0,006 & 0,224 \\
\hline Deportiva & 0,008 & $0,011^{* *}$ \\
\hline Naturaleza & $-0,007$ & $0,054^{*}$ \\
\hline Mar & $-0,002$ & 0,647 \\
\hline \multicolumn{3}{|l|}{ Lealtad al destino } \\
\hline Satisfacción & $-0,003$ & 0,547 \\
\hline Calificar experiencia & $-0,019$ & $0,011^{* *}$ \\
\hline Repetir viaje & 0,010 & $0,027^{* *}$ \\
\hline Recomendar destino & 0,005 & 0,374 \\
\hline (Constante) & $-0,127$ & 0,544 \\
\hline
\end{tabular}

Fuente: Elaboración propia en base a los resultados de los análisis de regresión realizados

Los resultados indican que las variables demográficas son las más importantes para establecer por qué los turistas eligen Canarias como destino gastronómico. A mayor edad, nivel educativo e ingresos, mayor es la probabilidad de que el turista elija el destino por este motivo. Sin embargo, cuantas más personas vivan en el hogar del turista, menor es la probabilidad de que viaje para disfrutar la gastronomía canaria. Además, a pesar de que en las tablas de contingencia mostró no tener una gran relevancia, la regresión lineal indica que los hombres (la variable "Sexo" toma el valor 0 ) son más propensos a elegir Canarias como destino gastronómico que las mujeres.

El análisis muestra también la gran relevancia de los motivos de elección del destino. Esto implica que lo turistas que eligen Canarias por su gastronomía no lo hacen únicamente por este motivo, sino que combinan su deseo de probar la gastronomía de las islas con otros atributos del destino que también desean disfrutar. Curiosamente, elegir el destino para practicar turismo de sol y playa no muestra ser significativo para el análisis. Probablemente porque, al ser el producto principal del archipiélago, la mayor parte de los turistas lo elijan por ello, tanto los que ven la gastronomía como un incentivo para visitar Canarias, como los que no. Llama la atención, que aquellos turistas que eligen el archipiélago por estar en Europa y por el precio del viaje son menos propensos a elegirlo por su gastronomía. Seguramente, estos turistas hayan elegido Canarias como destino por conveniencia en el momento de reservar sus vacaciones (cercanía, facilidad de acceso, precio...) y no por los atributos del mismo. 
Los turistas que eligen Canarias como destino de turismo gastronómico suelen conocer el destino a través de blogs o canales no especificados en la encuesta. Sin embargo, los que lo conocen a través de revistas de turismo o por anuncios publicitario televisivos, son menos propensos a visitar Canarias con este objetivo. Además, los turistas gastronómicos suelen permanecer en el destino por periodos más cortos y alojarse en establecimientos de mayor categoría. En lo relativo a los acompañantes con los que se realiza el viaje, esta variable no parece ser relevante para explicar por qué se elige Canarias como destino gastronómico. Tan solo los que viajan en pareja muestran una ligera tendencia a ser algo más propensos a elegir el destino por este motivo.

En cuanto al gasto incurrido para realizar el viaje, los turistas gastronómicos tienden a gastar más, en general, al viajar a Canarias que los turistas que no muestran estar motivados por la gastronomía del destino. En concreto, son más propensos a pagar más en el vuelo y, obviamente, en restaurantes. Sin embargo, gastan menos que otros turistas en practicar deportes y en comprar bienes inmuebles. Los turistas gastronómicos muestran ser más propensos a realizar actividades realizadas con la observación de estrellas (en Canarias, usualmente es combinado con la degustación de vino), el disfrute de la cultura tradicional, el consumo de productos gastronómicos y la realización de deporte (a pesar de ser menos propensos a gastar más dinero en practicar deportes). A pesar de ello, son menos propensos a visitar playas, piscinas, otras islas distintas a su destino principal, realizar excursiones organizadas, visitar museos, parques de ocio, practicar actividades de ocio y visitar espacios naturales.

Por último, resulta llamativo el hecho de que los turistas gastronómicos, a pesar de ser más propensos a valorar peor el viaje realizado a Canarias que otros turistas, tengan una tendencia mayor que otros segmentos a repetir su viaje a las islas.

\section{Discusión de Resultados y Conclusión}

El objetivo del presente estudio era llevar a cabo una caracterización del perfil del turista que elegía Islas Canarias para disfrutar de su gastronomía. Para ello, se acudió a la EGT elaborada por el ISTAC y se llevaron a cabo análisis mediante tablas de contingencia y regresión lineal. En base a los resultados, podemos afirmar que más de un 50\% de los turistas que visitan Islas Canarias consideraron la gastronomía como un factor que influyó "bastante" o "mucho" en su elección del destino. Además, se encontraron diferencias significativas en aspectos tales como la nacionalidad y otras variables sociodemográficas, así como las características del viaje en este segmento concreto de turistas gastronómicos.

El presente estudio presenta importantes contribuciones a la literatura sobre turismo gastronómico. En primer lugar, hemos resuelto la controversia planteada en cuanto a la importancia del segmento del turismo gastronómico, pues diversos estudios han dudado de la magnitud de dicho segmento (McKercher et al., 2008). Así, derivado de los resultados obtenidos, concluimos que el segmento de turistas motivados por la gastronomía sí tiene una importancia considerable con respecto al total de segmentos. En este sentido, podemos afirmar que nos posicionamos del lado de los investigadores que abogan por una clara importancia creciente de dicho segmento de mercado (Quan \& Wang, 2004). Además, hemos dado respuesta a la urgente necesidad planteada en la literatura previa en turismo gastronómico sobre la caracterización del perfil del turista que tiene la gastronomía como elemento motivacional del viaje (Chen \& Huang, 2016; Ryu \& Jang, 2006).

Sin embargo, nuestros resultados contradicen las investigaciones previas que mantenían que la gastronomía podía contribuir positivamente a la satisfacción del viaje (e.g., Berbel-Pineda et al., 2019; Du Rand \& Heath, 2006), pues la regresión lineal realizada indica que cuanto mayor sea la motivación por disfrutar de la gastronomía por parte de los turistas, menor tenderá a ser su valoración del viaje realizado. A pesar de ello, los turistas gastronómicos son más propensos a repetir sus viajes a Canarias que otros turistas. Esto podría explicar los resultados contradictorios a la literatura previa hallados, pues los turistas repetidores tienden a valorar peor los viajes realizados (Padrón-Ávila \& Hernández-Martín, 2019b).

Nuestro estudio también presenta importantes implicaciones prácticas que pueden ser utilizadas por los profesionales del turismo gastronómico, así como por los agentes encargados de llevar a cabo la promoción de destinos turísticos. La literatura académica sobre turismo gastronómico está de acuerdo en presentar los atributos gastronómicos del destino como un factor esencial en la promoción de destinos turísticos (e.g., Du Rand \& Heath, 2006). Y de hecho, una buena caracterización de la demanda a través del estudio del perfil del turista parece un buen punto de partida para empresas y destinos de cara a enfocar sus acciones de comunicación así como sus propuestas de valor a los clientes (Dorta-Afonso \& 
Cantero-García, 2018). Los resultados obtenidos en el presente estudio sugieren que la mejor forma de llevar a cabo acciones de comunicación por parte de organizaciones gestoras de destinos así como empresas de restauración serían los blogs y no tanto los canales tradicionales como la televisión o las revistas de turismo. Además, debido a que son turistas más interesados en los aspectos culturales, las acciones de comunicación de los destinos y empresas de restauración podrían ser llevadas a cabo conjuntamente con otro tipo de organizaciones culturales que pongan en valor la autenticidad del destino a través de la cultura en general y de la gastronomía en particular.

A pesar de las importantes contribuciones teóricas y prácticas que derivan de la presente investigación, nuestro estudio también presenta algunas limitaciones que merecen la atención y que abren interesantes líneas de investigación futuras. A pesar de que el tamaño de la muestra utilizado en los análisis es mucho mayor a la mayoría de estudios sobre turismo gastronómico (e.g., Berbel-Pineda et al., 2019), es cierto que el ámbito geográfico está limitado al de los turistas que han visitado las Islas Canarias. Por lo tanto, sería interesante que futuros estudios comprobasen si el perfil del turista gastronómico identificado aquí se corresponde con el de otros destinos diferentes. También, se podrían considerar diferencias dentro del propio destino Islas Canarias, como las diferentes islas o regiones, para comprobar si el perfil del turista motivado por la gastronomía difiere en función de la zona visitada. Además, aunque se ha señalado en la literatura la poca estacionalidad del turismo gastronómico desde el punto de vista de la oferta (Hjalager \& Corigliano, 2000), en este estudio se ha seguido la premisa y no se ha tenido en cuenta el momento del año en el que los turistas visitaban el destino. En este sentido, sería interesante comprobar si existen diferencias en los turistas motivados por gastronomía en los diferentes trimestres del año para concluir si la demanda tiene algún tipo de característica diferenciadora en función de la estacionalidad. Por último, hay que resaltar la índole transversal del análisis que se ha llevado a cabo, que si bien arroja luz sobre la situación actual del perfil del turista que visita Islas Canarias motivado por la gastronomía, no ofrece información sobre la evolución de las características diferenciales. Así, se propone que en un futuro se planteen diseños longitudinales con el fin de identificar las tendencias en la evolución de las características diferenciales del perfil del turista motivado por la gastronomía.

Como conclusión, planteamos que el segmento de mercado de los turistas motivados por la gastronomía tiene un tamaño considerable en Canarias, así como unas características particulares que lo diferencian del resto. Empresas y destinos que quieran centrarse en este segmento deberían tener en cuenta el perfil del turista gastronómico de cara a adaptar tanto sus propuestas de valor como sus acciones de comunicación al mismo. Además, el estudio realizado es relevante para dar a conocer a los gestores turísticos de Canarias la necesidad de mejorar la satisfacción de los turistas gastronómicos con el viaje al archipiélago, pues los resultados indican que son más propensos a gastar más en el destino que otros turistas. Esto podría ayudar, a su vez, a incrementar las probabilidades de que recomienden el destino a otros turistas gastronómicos. Para las empresas gastronómicas, este estudio les ayuda a conocer el perfil del turista más propenso a consumir en ellas, ayudándoles a diseñar campañas de promoción y a adecuar su producto al segmento de mercado al que se orientan.

\section{Bibliografía}

Berbel-Pineda, J. M., Palacios-Florencio, B., Ramírez-Hurtado, J. M. \& Santos-Roldán, L. (2019). Gastronomic experience as a factor of motivation in the tourist movements. International Journal of Gastronomy and Food Science, 18, 100171.

Chavarria, L. C. T. \& Phakdee-auksorn, P. (2017). Understanding international tourists' attitudes towards street food in Phuket, Thailand. Tourism Management Perspectives, 21, 66-73.

Chen, Q. \& Huang, R. (2016). Understanding the importance of food tourism to Chongqing, China. Journal of Vacation Marketing, 22, 42-54.

Cohen, E. \& Avieli, N. (2004). Food in tourism: Attraction and impediment. Annals of tourism Research, 31, 755-778.

Dorta-Afonso, D. \& Cantero-García, M. (2018). Adaptación al español de la escala NES: segmentación de turistas en base a motivaciones ambientales.

Dorta-Afonso, D. \& Hernández-Martín, R. (2015). Subnational tourism competitiveness performance. The Canary Islands vs. The German Länder. European Journal of Tourism Research, 10, 51-63.

Du Rand, G. E. \& Heath, E. (2006). Towards a framework for food tourism as an element of destination marketing. Current issues in tourism, 9, 206-234. 
Dwyer, L. \& Kim, C. (2003). Destination competitiveness: Determinants and indicators. Current issues in tourism, 6(5), 369-414.

Hall, C. M., Sharples, L., Mitchell, R., Macionis, N. \& Cambourne, B. (2004). Food tourism around the world. Routledge.

Hjalager, A. \& Corigliano, M. A. (2000). Food for tourists-Determinants of an image. International journal of tourism research, 2, 281-293.

ISTAC. (2018). Encuesta sobre el Gasto Turístico. Metodología 2018. Recuperado de http://www.gobiernodecanarias.org/istac/galerias/documentos/C00028A/2018/EGT-2018-metodologia.pdf

Kim, S., Park, E. \& Lamb, D. (2019). Extraordinary or ordinary? Food tourism motivations of Japanese domestic noodle tourists. Tourism Management Perspectives, 29, 176-186.

Kim, Y. H., Goh, B. K. \& Yuan, J. (2010). Development of a multi-dimensional scale for measuring food tourist motivations. Journal of Quality Assurance in Hospitality \& Tourism, 11, 56-71.

Lin, L. \& Mao, P.-C. (2015). Food for memories and culture-A content analysis study of food specialties and souvenirs. Journal of Hospitality and Tourism Management, 22, 19-29.

López-Guzmán, T., Uribe Lotero, C. P., Perez Galvez, J. C. \& Rios Rivera, I. (2017). Gastronomic festivals: Attitude, motivation and satisfaction of the tourist. British Food Journal, 119, 267-283.

Mason, M. C. \& Paggiaro, A. (2012). Investigating the role of festivalscape in culinary tourism: The case of food and wine events. Tourism management, 33, 1329-1336.

McKercher, B., Okumus, F. \& Okumus, B. (2008). Food tourism as a viable market segment: It's all how you cook the numbers! Journal of Travel \& Tourism Marketing, 25, 137-148.

Padrón-Ávila, H. \& Hernández-Martín, R. (2017). Tourist points of interest: Analytical relevance, methodological proposal and study case. PASOS: Revista de Turismo y Patrimonio Cultural, 15(4), 979-1000.

Padrón-Ávila, H. \& Hernández-Martín, R. (2019a). Preventing Overtourism by Identifying the Determinants of Tourists' Choice of Attractions. Sustainability, 11, 5177.

Padrón-Ávila, H. \& Hernández-Martín, R. (2019b). Why do tourists differ in their likelihood to visit attractions? The case of Lanzarote. International Journal of Tourism Research.

Quan, S. \& Wang, N. (2004). Towards a structural model of the tourist experience: An illustration from food experiences in tourism. Tourism management, 25, 297-305.

Rasoolimanesh, S. M., Ali, F. \& Jaafar, M. (2018). Modeling residents' perceptions of tourism development: Linear versus non-linear models. Journal of Destination Marketing \& Management, 10, 1-9.

Ritchie, J. B. \& Crouch, G. I. (2003). The competitive destination: A sustainable tourism perspective. Cabi.

Ryu, K. \& Jang, S. (2006). Intention to experience local cuisine in a travel destination: The modified theory of reasoned action. Journal of Hospitality \& Tourism Research, 30, 507-516.

Sánchez-Cañizares, S. M. \& López-Guzmán, T. (2012). Gastronomy as a tourism resource: Profile of the culinary tourist. Current issues in tourism, 15, 229-245.

Tikkanen, I. (2007). Maslow's hierarchy and food tourism in Finland: Five cases. British food journal, 109, 721-734.

Wang, N. (1999). Rethinking authenticity in tourism experience. Annals of tourism research, 26, 349-370.

Wijaya, S., King, B., Nguyen, T.-H. \& Morrison, A. (2013). International visitor dining experiences: A conceptual framework. Journal of Hospitality and Tourism Management, 20, 34-42. 